2017-07-31

\title{
An SDN-approach for QoE management of multimedia services using resource allocation
}

\section{Barakabitze, Alcardo Alex}

http://hdl.handle.net/10026.1/12531

\subsection{9/ICC.2017.7997261}

978-1-4673-8999-0

IEEE

All content in PEARL is protected by copyright law. Author manuscripts are made available in accordance with publisher policies. Please cite only the published version using the details provided on the item record or document. In the absence of an open licence (e.g. Creative Commons), permissions for further reuse of content should be sought from the publisher or author. 


\title{
An SDN-approach for QoE management of multimedia services using resource allocation
}

\author{
Elisavet Grigoriou*, Alcardo Alex Barakabitze ${ }^{\dagger}$, Luigi Atzori*, Lingfen Sun ${ }^{\dagger}$ and Virginia Pilloni* \\ * University of Cagliari, Cagliari, Italy \\ \{elisavet.grigoriou, l.atzori, virginia.pilloni\}@ diee.unica.it \\ $\dagger$ University of Plymouth, Plymouth, UK \\ \{alcardoalex.barakabitze, L.Sun\}@plymouth.ac.uk
}

\begin{abstract}
Future networks will be accompanied by new heterogeneous requirements in terms of end-users Quality of Experience $(\mathrm{QoE})$ due to the increasing number of application scenarios being deployed. Network softwarization technologies such as Software Defined Networks (SDNs) and Network Function Virtualization (NFV) promise to provide these capabilities. In this paper, a novel QoE-driven resource allocation mechanism is proposed to dynamically assign tasks to virtual network nodes in order to achieve an optimized end-to-end quality. The aim is to find the best combination of network node functions that can provide an optimized level of $\mathrm{QOE}$ to the end users though node cooperation. The service in question is divided in tasks and the neighbor nodes negotiate the assignment of these considering the final quality. In the paper we specifically focus on the video streaming service. We also show that the agility provided by SDN/NFV is a key factor for enhancing video quality, resource allocation and $\mathrm{QoE}$ management in future networks. Preliminary results based on the Mininet network emulator and the OpenDaylight controller have shown that our approach can significantly improve the quality of a transmitted video by selecting the best path with normalized QoS values.
\end{abstract}

Index Terms-SDN, NFV, Multimedia Services, resource allocation, video quality, QoE.

\section{INTRODUCTION}

Future networks will rely on heterogeneous wireless and wired physical infrastructures and a significant amount of multimedia traffic. The expected explosion of traffic will lead to inefficient utilization of resources which necessitates future network resources to be unified and dynamically pooled as well as offered in as-a-Service fashion to multiple end-users. Furthermore, issues such as lack of flexibility and agility are going to be observed, especially in case of new multimedia services such as teleconference, telemedicine, etc. In today's competitive environment, users have the option to choose from a variety of Service Providers (SPs). Therefore, the service availability is not enough anymore. Thus, SPs must deliver their services in such a way that users will fully enjoy a rich experience at a logical price with an improved Quality of Experience (QoE).

The Qualinet defines QoE as [1]: "Quality of Experience $(Q o E)$ is the degree of delight or annoyance of the user of an application or service. It results from the fulfilment of his or her expectations with respect to the utility and or enjoyment of the application or service in the light of the user's personality and current state." Thus, QoE is defined as the measure of how well a system or an application meets the user's expectations. This is different from Quality of Service (QoS), which is focusing on measuring performance from a network perspective. With no doubt, QoE is directly related to QoS. Considering the multimedia engineering problems (e.g. processing, compression) in traditional networks, the challenge for a service provider is to have the right set of QoE control and management mechanisms to have a greater impact on its balance sheets that would result into reducing the users' churn. According to Qualinet [1], the QoE is influenced by many factors which can be categorized into three groups namely, Human (e.g., related to demographic characteristics), System (e.g., related to technical quality characteristics of an application/service) and Context (e.g., related to user's environment). Considering the bandwidth increase which is expected in future networks such as $5 \mathrm{G}$ networks in conjunction with the rapid increase of multimedia services, it is evident that, the end users demand for even higher quality media content will also increase rapidly.

To this end, it is likely that, new technologies that could provide an anticipating resource management and efficient QoE provisioning mechanisms to the end users are required in future networks. Software Defined Network (SDN) is a promising and a natural choice for achieving such future network capabilities. In SDNs, the Control Plane is separated from the Data Plane. This makes it easier for network resources management in terms of design, delivery and operation of network services in a scalable, flexible and dynamic way. Therefore, automation, programmability and network control can be achieved to provide flexible and scalable networks. Furthermore, Network Function Virtualization (NFV) is envisaged to allow virtualization of software-based network functions by breaking the monolithic approach to functional software and hardware that exists in today's vendor offerings. There is no longer a need to install and manage dedicated hardware devices for networking and service functions. For network operators, SDNs and NFV have the potential for significant reductions of CAPEX and OPEX, with an aim on softwarization of everything everywhere in order to meet various network management and service provisioning objectives such as flexibility and scalability [2] [3].

Network softwarization using SDN and NFV enables distributed virtual platforms to execute any network functions 
and networked services as applications on Virtual Machines (VMs) which are allocated, managed and moved dynamically on general purpose hardware. SDN and NFV are considered as a key enabling technology and an appealing solution in future networks such as $5 \mathrm{G}$ for the value added QoE-driven services provisioning such as high quality $3 \mathrm{D} / 4 \mathrm{~K} / 8 \mathrm{~K}$ video and high bitrate contents distribution across the network coupled with personalized services interactivity.

Motivated by the incentives and opportunities provided by these technologies, this paper presents an SDN-based approach for QoE management of future multimedia services through QoE-driven network path assignment. We apply the task-level scheduling with the intention of assigning tasks related to multimedia application to network elements. To achieve this goal, we developed a QoE-driven dynamic task allocation scheme for adaptive video streaming over SDN/NFV enabled networks. After all, we want to be able to deploy new applications, services and infrastructures in order to meet future changing business goals while succeeding high level of endusers' QoE. This is the reason why, we are using network softwarization technologies such as SDN and NFV.

The rest of this paper is organized as follows: Section II describes the state-of-the-art focusing on optimization of network resource allocation and QoS/QoE-driven dynamic routing using SDN. Section III presents our problem formulation along with the task and network model. Section IV presents our task assignment model in conjunction with the theoretical description of the task assignment algorithm. We present our experimental setup and the performance evaluation of our testbed in Section VI and VII respectively before concluding our remarks in Section VIII.

\section{RELATED WORK}

The network resource allocation is a major problem in multimedia communications. There have been many studies in recent years tackling this problem. For example, [4] addressed the issue regarding optimization of network resource allocation for wireless video delivery. Focusing on throughput maximization, a cross-layer QoE-based optimization framework was proposed in [5] to allocate network resources efficiently for a video delivery service. In real-time applications such as video streaming, there are dynamic changes of the application requirements (e.g. delay, packet loss, etc.). Note that, initially, the QoE-based optimization solutions were proposed for elastic application such as file transfers, which captures the user's satisfaction as a function of data rate using a concave utility function [6]. The aim from network operator's view is to allow the maximum number of users to join the system and in the same time to keep a good level of service quality. Their technique is based on the fact that, the user is choosing a charge per unit time and the network is determining allocated rates. But must exist a balance between them in order to have an optimized system.

In [7], a framework is proposed which applies dynamic traffic shaping on home network gateway based on network traffic statistics and monitoring of video flow to achieve dynamic allocation of bandwidth for each video flow in real-time. The authors in [8] present an SDN-testbed along with an SDN-based video streaming architecture for monitoring the streaming flows in real time using MPLS protocol to change the routing paths. But that approach is designed only from video service provider perspective. In [9], the architecture that integrates the SDN and NFV is described along with the mandatory requirements of adopting new technologies in mobile networks. Authors demonstrate the feasibility of SDN and NFV technologies for future networks such as 5G. But as the authors point out, robustness and reliability are not provided. In order to proceed with our work, we considered the work from [10]. By leveraging the NFV and $\mathrm{SDN}$ in order to solve the challenges of resource, traffic and mobility management in the current mobile networks such as 4G/LTE networks, new concepts and opportunities for the Software Defined Mobile Network (SDMN) architecture is analyzed theoretically and experimentally in [10] to meet the end user's traffic requirements. In [11], authors demonstrate an SDN/NFV enabled network domain approach towards providing an agile video transcoding process for maintaining the QoE level of a media service when network congestion occurs. This approach adopts the encoding characteristics provisioned video stream to implements a self-optimization and self-adaptation VNF of real-time video streams.

\section{Problem Formulation}

At the network level, in the traditional systems, all media flows from the server to clients follow the same network path while it is the case that, such a path might not be the optimal one for all types of media flows [12]. Thus, it is required to develop mechanisms that allow delivering each user media flow over the "best available" path using the "best service configuration" to maximize the end users' QoE. To achieve this, our approach aims at providing the QoEcentric traffic flow control and routing mechanisms, a concept which is motivated by the Economic Traffic Management presented in [13]. The QoE-centric traffic flow control and routing mechanisms aim to enable several network elements to cooperate in measuring and collection of the QoE influencing factors in the SDN network.

In this paper, our target is to optimize the overall QoE in case of adaptive video streaming application using NFV and SDN technologies. The quality optimization problem consists of maximizing a quality function. We formulate the problem of dynamic task allocation in an adaptive video streaming scenario considering QoE influence factors in order to improve the overall QoE. To achieve this, we first propose a task model and a network model, in the following subsections.

\section{A. Task Model}

Our task model considers, as an example, a video streaming service which includes four tasks: caching (original source video), encoding, forwarding (it refers to one forwarding 
action running on one node) and playing back (client side). A service can be decomposed into a set of tasks described as a Directed Acyclic Graph (DAG) of tasks denoted as: $G_{T}=\left(T, E_{T}\right) . T=\{1, \ldots, \lambda, \ldots, \Lambda\}$ represents the set of tasks and $E_{T}=\left(e_{v w}\right)$ is the set of edges such that each edge $e_{v w}$ represents a unidirectional data transfer from task $v$ to task $w$. Figure 1 illustrates an example of a general serial DAG where each node represents a task. A binary vector $X_{i}=\left[x_{i \lambda}\right]$ for

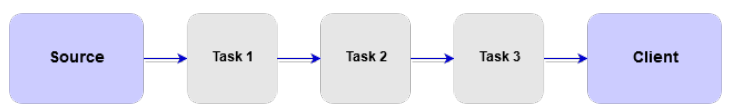

Figure 1. Example of a general serial DAG.

$\lambda \in T$, can be assigned to each node $i$ in the network. $x_{i \lambda}$ is a boolean value representing the current state of the node $i$ corresponding to task $\lambda$. When node $i$ performs a task $\lambda$ then $x_{i \lambda}=1$. Figure 2 illustrates the DAG of the media streaming service task chain. Thus, each one of these tasks should be executed in order to deliver the video from the media server to the end-user. Depending on the network and application parameters, our goal is to improve/optimize the overall QoE by making the best task assignment to network nodes.

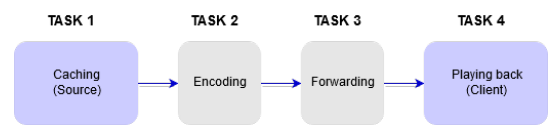

Figure 2. DAG for adaptive media streaming

\section{B. Network Model}

The network is modeled as a DAG $G_{Z}=\left(Z, E_{Z}\right)$. The vertices represent the nodes $Z=\{1, \ldots, i, . ., N\}$ and the links are described by the set of edges $E_{Z}=\left(e_{i j}\right)$. Each node of the DAG is a Network Element (NE) which can be based on NFV where each NFV includes many VMs (e.g., for storage and network). Figure 3 illustrates the network model which depicts the SDN/NFV overview with the following components:

1) Hardware Resources which consist of Compute, Storage and Network modules. These are the physical resources related to CPU, memory and network, respectively.

2) Virtualization Layer abstracts the hardware resources and anchors the VNFs (Virtual Network Functions) to the virtualized infrastructure.

3) Virtual Resources consist of the vCompute, vStorage and vNetwork modules. The Data Plane includes the VNFs which are controlled by the SDN-Controller via Southbound API using the OpenFlow protocol. The SDNController communicates with a "QoE management application "in the Management Plane via a Northbound API, in order to monitor and manage the media flows in the Data Plane.

Different services have different requirements and different parameters. Depending on user's requirements, each service can be divided into smaller tasks that can be assigned to

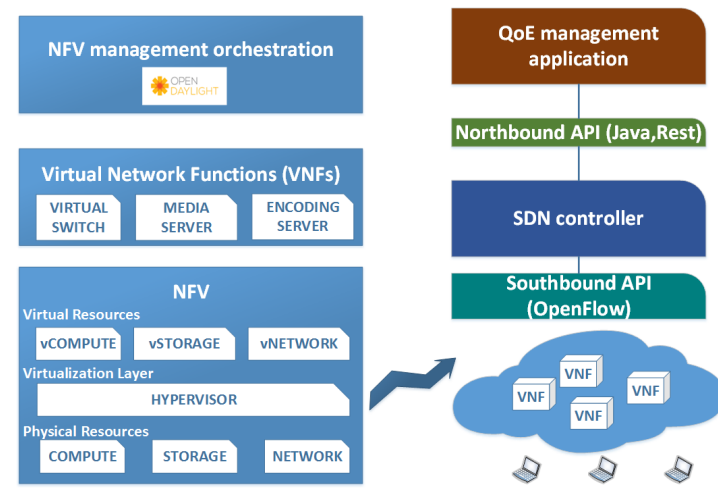

Figure 3. Network Model NFV/SDN view

different NEs (namely different VNFs) in order to deliver the video to the end-user using an Openflow-based virtual switch from a media server where the original version of the video is stored.

\section{TASK ASSIGNMENT MODEL}

The aim of optimization problem is to find the best path of nodes in order to improve the overall QoE. Although, this is an NP-hard problem, which could be time and energy consuming [14], we apply a centralized optimization approach trying to make the best task assignment in order to improve QoE. A centralized optimization problem is defined, where a utility function $u_{n e t}$ is assigned to a network for a given strategy vector $x_{i}$. The goal of the network is to maximize its own utility. Our solution chooses a task assignment strategy $x_{i}$ that maximizes utility function. Therefore, a strategy $x_{i}^{*}$ is preferred to a strategy $x_{i}$, if and only if $u_{n e t}\left(x_{i}^{*}\right)>u_{n e t}\left(x_{i}\right)$.

\section{A. Overall Utility Function}

In order to formalize the correlation between network performance and user perceived quality, a utility function is defined. The concept of utility function is adopted from economics which provides the means for reflecting a normalized and transparent way of various services performance prerequisites, users degree of satisfaction, different types of networks diverse resources and dissimilar QoS provisioning mechanisms and capabilities under common utility-based optimization problems [15]. Our algorithm decides which particular NE should execute a given task $\lambda$ by maximizing network utility function.

The overall utility function consists of both the benefit and the cost for a node $i \in Z$ executing a task $\lambda \in T$. The objective function is defined as:

$$
u_{n e t}=\max \sum_{i \in Z} \sum_{\lambda \in T}\left(\alpha \times b_{i \lambda}-\beta \times c_{i \lambda}\right) \times x_{i \lambda}
$$

where $\alpha, \beta$ are the weighting factors. $x_{i \lambda}$ is a boolean variable that can be 0,1 depending if a node $i$ executes a task $\lambda . b_{i \lambda}$ is related to the benefit that exists, if a task $\lambda$ is executed in node $i$. $c_{i \lambda}$ refers to the cost for node $i$ running task $\lambda$. It is defined as the cost for resource consumption of both CPU and 
memory, i.e. cost $=f(C P U$, memory $)$ and can be calculated as follows [16]:

$$
c_{i \lambda}=\gamma_{i} \times C P U_{i \lambda}+\delta_{i} \times \text { Memory }_{i \lambda}, \forall i \in Z, \forall \lambda \in T
$$

where $\gamma, \delta$ are scale factors related to node $i$ which allow us to weight the cost according to the required CPU and memory for a particular task in node $i$ e.g. a task such as "encoding" needs more CPU and memory than a "forwarding" task. These weights depend on node $i$. Furthermore, the benefit is related to QoS level regarding to delay, jitter, bandwidth and packet loss. $b_{i \lambda}$ is defined as the execution benefit for running task $\lambda$ at node $i$. A correlation model from [17] is used to map the QoS parameters to a QoE metric for video streaming service. The model is derived by a normalized QoS value as follows:

$$
b_{i \lambda}=Q_{r} \times(1-Q o S(C))^{\frac{\operatorname{QoS}(C) \times A}{R}}
$$

where $A$ is a constant relating to the video resolution class such as Standard Definition (SD) $(A=120)$ or High Definition (HD) $(A=240)$. If the subscribed service class is high, the constant $A$ is assigned to a higher value. It means that the $\mathrm{QoE}$ level which the premium service subscriber's requests is higher than normal service subscribers's in the network condition of the same QoS level. $R$ is a constant which reflects the structure of the video frames according to the GoP (Group of Picture) length and it is defined as $R=24$. $Q_{r}$ is a constant factor that determines the overall QoE of video streaming service. Based on literature [17], the constant $Q_{r}$ is set to $=0.95$.

The normalized QoS value $(Q o S(C))$ refers to the network performance and is calculated using Eq. 4. The $Q o S(C)$ value can be simply calculated with the total sum of the values multiplying the measured QoS parameters in network layer with the allocated weights. These weights are selected according to the type of the access network for the service. The considered QoS parameters are Packet Loss (PL), Packet Jitter (PJ), Packet Delay (PD) and Bandwidth (BW). The normalized QoS value reflects the network condition and is calculated as follows [17]:

$Q o S(C)=P L \times W_{P L}+P J \times W_{P J}+P D \times W_{P D}+B \times W_{B W}$

where $C=\{1 \ldots, i . ., N\}$ is a sub-set of $Z$, a set of nodes involving in video delivery from the media server to the client. $W_{P L}, W_{P J}, W_{P D}$ and $W_{B W}$ are the weights for packet loss, packet jitter, packet delay and bandwidth, respectively. Note that, the weights of QoS parameters are assigned according to the quality standard bounds and their relative importance degree are given from [17] as follows, PL 58,9\%, PJ 15.1\%, PD $14,9 \%$ and BW 11,1\%. The weight of QoS parameters is assigned based on the quality standard bounds recommended in the standardization organizations (e.g. ITU-T) and its relative importance degree. The objective function is subjected to the following constraints.

Constraint 1 Every task $\lambda$ must be executed in at least one node such that

$$
\sum_{i \in Z} x_{i \lambda} \geq 1 \forall \lambda \in T
$$

Constraint 2 Each node can execute only one task at a time

$$
\sum_{\lambda \in T} x_{i \lambda}=1 \forall i \in Z
$$

Constraint 3 If node $i$ is executing task $\lambda$ then node $j$ that is going to execute task $(\lambda+1)$ (next task), must have a relation (link) with node $i$ of $e_{i j}=1$

$$
e_{i j}=\left\{\begin{array}{ll}
1 & \text { if } x_{i, \lambda}=1 \text { AND } x_{j,(\lambda+1)}=1 \\
0 & \text { otherwise }
\end{array} \forall i, j \in Z\right.
$$

Constraint 4 Each network element has specific available resources and every task requires specific amount of resources. Thus, the available resources for each network element cannot be less than the required amount of resources.

For node $i \in Z$, we define a set of available resources as Available $_{i}=\left\{C P U_{i}\right.$, Memory $\left._{i}, \ldots\right\}$

For a task $\lambda \in T$, we define a set of required resources as Required $_{\lambda}=\left\{C P U_{\lambda}\right.$, Memory $\left._{\lambda}, \ldots\right\}$

$$
\text { Required }_{\lambda} \leq \text { Available }_{i}, \forall \lambda \in T, \forall i \in Z
$$

\section{TASK ASSIGNMENT ALGORITHM}

Using the network topology shown in Figure 4, our algorithm finds which is the best path to deliver the video, while executing all the defined tasks. Every NE has specific available resources and every task requires a specific amount of resources regarding to the amount of CPU and memory. The resource allocation algorithm employs the following steps:

1) Based on network topology, the algorithm finds all the paths that can be used to deliver the video from each media server to each client and creates a list of them.

2) Based on the proposed network model and task model and the previous list, it creates a new list with all possible paths considering all the constraints. Moreover, every path must starts from a "media serve" node and ends with a "client" node and must includes nodes that execute all the tasks.

3) For every path, it calculates the $\operatorname{QoS}(C)$ value of the path based on Eq. 4. Since every link of the path in our topology has different delay and bandwidth, the algorithm considers the average delay and average bandwidth of the path.

4) For every path, then it calculates the "Benefit of the path" based on Eq. 3 by considering the $Q o S(C)$ value of a path, $Q_{r}$ (a factor which determines the overall $\mathrm{QoE}$ of the video streaming), the resolution class $A$ and the structure of the video frames $R$.

5) For every path, it calculates the "Cost of a path" based on Eq. 2 by considering the required amount of CPU and memory of a task $\lambda$.

6) The algorithm calculates the $u_{\text {net }}$ function, based on Eq. 1 using the "Benefit of the path" and the "Cost of a path".

7) The algorithm will use the path with the highest $u_{n e t}$ value to deliver the video to the Client. 


\section{EXPERIMENTAL SETUP}

An experimental testbed was setup based on a network emulator, Mininet [18] and an SDN-Controller implemented by OpenDaylight [19]. The testbed consists of 2 video streaming servers, 3 clients, 7 virtual switches and the SDN controller, as illustrated in Figure 4. The network access was provided by using a Cisco Linksys x1000 device compatible with IEEE $802.11 \mathrm{~b} / \mathrm{g} / \mathrm{n}$ operating at $2.4 \mathrm{GHz}$ bandwidth. Mininet was installed in a Toshiba computer with Intel ${ }^{\circledR}$ Core $^{\mathrm{TM}}$, i7-3770 CPU@ $3.40 \mathrm{GHz}, 16 \mathrm{~GB}$ of RAM installed with Linux Ubuntu 14.04, 64 bit. The SDN-controller Ethernet port was fixed to a static IP address to ensure service availability throughout the experimentation period. For simplicity, during network path

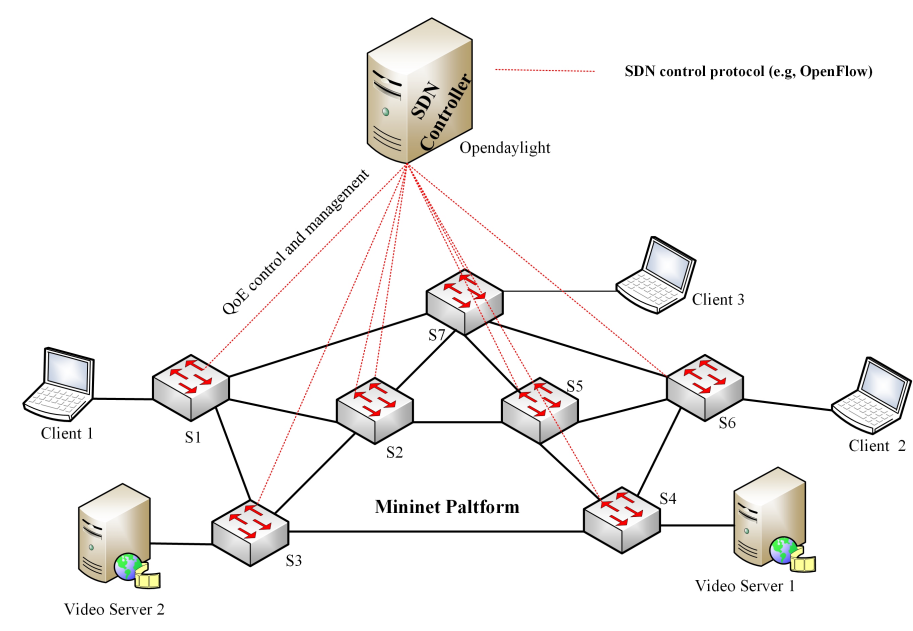

Figure 4. Experimental Setup

assignment to a particular multimedia traffic/session flow, our approach first specifies the particular network path links and nodes by utilizing an automatic optimal path configuration of each traffic/session flows to be established through the assigned path loss probability and an average end-to-end delay of the link. During multimedia service flow establishment between a client and a video server, each of the following components performs the following functions [20]:

1) End-user clients initiate an SDN application request to the video server with their preferences and requirements such as video and screen resolution as well as the supported codecs of their devices.

2) Upon receiving a request, the video server (s) communicates these clients' requests to an SDN application with the parameter matching function. It also conveys client's information and requested parameters such as required video resolution, video bit rates and codecs.

3) An SDN application then determines the required parameters to be delivered to clients when there is a match received from the video server.

4) The associated multimedia traffic parameters are then sent by the SDN application to the SDN-Controller. Such parameters include the video codecs and video bit rates along with the required QoE model.
5) The SDN-Controller through the OpenFlow [21] performs the QoE-centric multimedia traffic flow control and routing mechanisms to determine the possible path that will maximize the QoE of the end-users requests.

In fact, when scalability and interoperability become the core requirements, we are able to create a generic solution using OpenFlow which should work across different service provisioning scenarios ranging from a multitude of vendors and ISPs.

\section{ViI. Performance Evaluation}

The objective of our experiments is to perform the evaluation of the QoE level provided by the proposed task allocation algorithm with respect to scalability and clients differentiation, bandwidth fluctuations and end-to-end delay variations. The programmability and the overall combination of features provided by SDN and NFV as well as the openness of OpenFlow [21] enabled us to have a fully realization of the real world networks throughout our experiments. Our experiments employ the VLC media player [22] for video streaming while for video stream delivery from any of the video server nodes to clients; the UDP/RTP protocols are used. The total available bandwidth to be accessible to video streams is set on different links to fluctuate between $20 \mathrm{~kb} / \mathrm{s}$ to $20 \mathrm{Mb} / \mathrm{s}$. Such bandwidth limit is motivated by the Wi-Fi routers using the wireless-A and wireless-G standards which can limit connection speeds with ISPs that offer $25 \mathrm{Mb} / \mathrm{s}$ for fast connections. As shown in Table 1, the resolutions of video streams to be delivered to clients were selected randomly between $360 \mathrm{p}, 720 \mathrm{p}$ and $1080 \mathrm{p}$ in the beginning of the experiments which was defined with the duration of 10 minutes and three clients selected randomly to receive a video from video server 1 . In order to evaluate our approach, the reference video file of an animated film called "Big Buck Bunny" which is widely used by researchers in the area of adaptive content distribution was selected. The uncompressed YUV video files in 360p, 720p and 1080p resolution were then encoded using the H.264 codec. Three different tests were carried out to evaluate the performance of our approach. The first test evaluates the bandwidth fluctuations and delay variations from a video server to a certain number of receiving clients. In the second test, we conducted two different experiments to evaluate the effects of packet loss on video quality at different delay variations. In experiment 1 , the delay was varied in the interval [20ms, 60ms] while in experiment 2 , the delay was varied in the interval $[10 \mathrm{~ms}, 30 \mathrm{~ms}]$. The available bandwidth in these two experiments was set to $1000 \mathrm{kbps}$ whereas the average packet loss probability was selected randomly in the interval $[0 \%, 20 \%]$. The last test evaluates the transmitted video quality as measured by the normalized QoS using Eq. 4. The network QoS parameters (packet loss rate, jitter, delay and bandwidth) which are related also with video quality were configured using netem [23] which is the well-known routing and traffic control feature for system monitoring, traffic classification and traffic manipulation. 
Table 1

RESOLUTIONS OF VIDEO STREAMS.

\begin{tabular}{|l|l|}
\hline Video resolution & Video bitrate $(\mathrm{kbps})$ \\
\hline $1080 \mathrm{p}$ & $100,200,600,1000,2000,4000,6000$, \\
& 8000 \\
$720 \mathrm{p}$ & $100,200,400,600,800,1000,1500,2000$ \\
$360 \mathrm{p}$ & $100,200,400,600,800,1000$ \\
\hline
\end{tabular}

\section{A. Bandwidth and end-to-end delay variations}

Figure 5 shows the relationship between the number of video servers that can serve a specified number of clients at varying link bandwidth and delay variation values. In practice, a change in the shared bandwidth will lead to a network resource reallocation process which is basically instructed by the resource allocation function. Every change in video quality is accounted for in the end user's QoE while the increasing number of quality fluctuations is believed to be impacted by the number of delay variations and packet loss rate on the network links.

We observe that, as the number of video delivery nodes

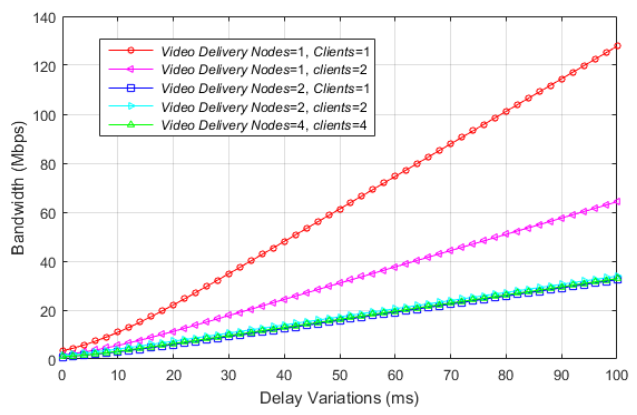

Figure 5. Bandwidth and delay variations with different number of clients and video servers.

increase, the delay variations on the network links have less effect on the available bandwidth required for transmitting videos to clients. For example, at $60 \mathrm{~ms}$ delay variation, 2 or 4 video servers can provide services to 1,2 or 4 clients at the same bandwidth of 20Mbps. Such observation has also an implication of delivering high videos quality to clients.

\section{B. Effects of Packet Loss variations on video quality}

As multimedia applications over IP networks continue to gain popularity, resource allocations with respect to multi-tier topology, user data sharing and cross-domain policies to be implemented using SDN approach continue to face a challenge as well [24]. Considering the media streaming services in a dynamic and heterogeneous applications in future networks such as $5 \mathrm{G}$, our approach differ from the conventional designs in the sense that, we model the utility and assignment of tasks to network nodes in order to improve the overall QoE level. Such design enables several network elements to cooperate during the process of QoE measurements and collection of the QoE influencing factors in the SDN platform. In order to do that, we conducted two different experiments to evaluate

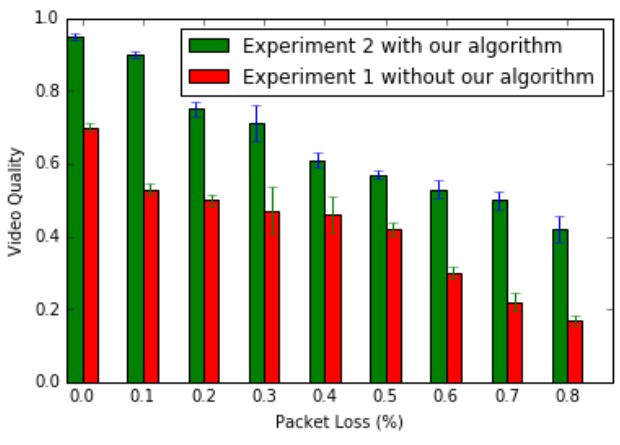

Figure 6. QoE values at different variations of packet loss and delay

the effects of packet loss on video quality at different delay variations. In experiment 1 , the delay was varied in the interval [20ms, 60ms], while in experiment 2 , the delay was varied in the interval [10ms, 30ms]. The average packet loss probability is selected randomly in the interval [0\%, 20\%]. The available bandwidth of $1000 \mathrm{kbps}$ was selected based on the fact that, $1 \mathrm{Mbps}$ was fairly enough for our experimentation taking into account that the aim was to investigate how delay and packet loss affect the transmitted video quality using our approach. Figure 6 shows the video quality of transmitted videos as the function of packet loss rate. As expected, we observe from Figure 6 that, as packet loss decreases, the video quality increases as indicated by the QoE values from the correlation model described in [17].

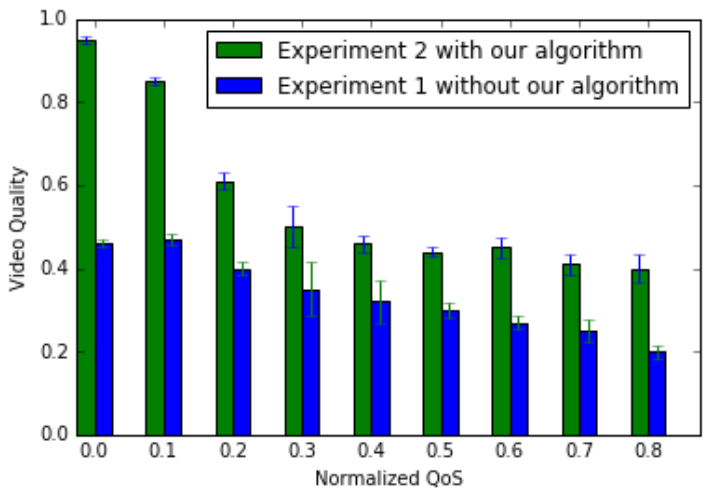

Figure 7. Transmitted video QoE with the normalized QoS values.

\section{Transmitted video $Q o E$ with the normalized $Q o S$ values}

Figure 7 shows the results of the transmitted video QoE without and with our proposed QoE-driven resource allocation algorithm. In this experiment, the normalized $Q o S(C)$ was calculated based on Eq. 4 where the constants $A$ and $R$ in Eq. 3 were assigned to 240 and 24 respectively by considering the used codec, network parameters set and video resolution. The blue color bar demonstrates the test experiment without using our proposed algorithm. It is evident that, the video quality increases as the normalized QoS value decreases. The video quality and the $Q o S(C)$ reflects the QoE metric for video 
streaming services and the network parameters/conditions respectively which are set as described in the second test done in the previous subsection B. Using the QoS-to-QoE correlation model in [17], our proposed approach can significantly improve the video quality at the normalized QoS values.

\section{CONCLUSION}

Although SDN and NFV promise new opportunities, a unified approach for leveraging these network softwarization technologies in the wireless and mobile domain is lacking. This paper presents an SDN-based approach for QoE management through cooperation and information exchange among network elements which are involved in the service delivery chain (e.g., from the video delivery nodes to clients). This approach enables more efficient resource utilization and simplifies network management using the elasticity of SDN/NFV technologies. Our aim is to find and provide the best combination of network nodes that can cooperate during the execution of the defined task and in the same time to improve the overall QoE level of the end users. To achieve this, we developed a QoE-driven dynamic task allocation scheme for adaptive video streaming over SDN/NFV enabled networks. We have shown that, the agility provided by network softwarization infrastructures using SDN/NFV is a key factor for enhancing video quality, resource allocation and QoE management especially in future networks.In the future, we will replicate this study to archieve an efficient QoE control and management of network/system resources between multiple players in the network domain (mobile network operators, content providers and cloud/service providers.

\section{ACKNOWLEDGMENT}

This paper is part of a project that has received funding from the European Union's Horizon 2020 research and innovation programme under the Marie Skłodowska-Curie grant agreement No 643072.

\section{REFERENCES}

[1] K.Brunnström, S.A. Beker, K.De Moor, A. Dooms,S. Egger,... B. Lawlor, "Qualinet white paper on definitions of quality of experience," 2013.

[2] D. Kreutz, F. M. V. Ramos, P. E. Veríssimo, C. E. Rothenberg, S. Azodolmolky and S. Uhlig, "Software-Defined Networking: A Comprehensive Survey," in Proceedings of the IEEE, vol. 103, no. 1, pp. 14-76, Jan. 2015.

[3] A. Galis et al., "Softwarization of Future Networks and Services Programmable Enabled Networks as Next Generation Software Defined Networks," Future Networks and Services (SDN4FNS), 2013 IEEE SDN for, Trento, 2013, pp. 1-7.

[4] V. Tsibonis, L. Georgiadis and L. Tassiulas, "Exploiting wireless channel State information for throughput maximization," in IEEE Transactions on Information Theory, vol. 50, no. 11, pp. 2566-2582,2004.

[5] M. Shehada and S. Thakolsri and Z. Despotovic and W. Kellerer, "QoEbased Cross-Layer Optimization for video delivery in Long Term Evolution mobile networks," Wireless Personal Multimedia Communications (WPMC), 2011 14th International Symposium on, Brest, pp. 1-5, 2011.

[6] F. Kelly "Charging and rate control for elastic traffic.," Eur. Trans. Telecomm., vol.8, pp.33-37, 1997.

[7] R. M. Abuteir, A. Fladenmuller and O. Fourmaux, "SDN Based Architecture to Improve Video Streaming in Home Networks," 2016 IEEE 30th International Conference on Advanced Information Networking and Applications (AINA), Crans-Montana, 2016, pp. 220-226.
[8] H. Nam, K. H. Kim, J. Y. Kim and H. Schulzrinne, "Towards QoEaware video streaming using SDN," 2014 IEEE Global Communications Conference, Austin, TX, 2014, pp. 1317-1322.

[9] J. Costa-Requena, J. Llorente Santos, V. Ferrer Guasch, K. Ahokas,. Premsankar, S. Luukkainen, I. Ahmad, M. Liyanage, M. Ylianttila, O. Lopez Perez, M. Uriarte Itzazelaia, and E. Montes de Oca, ”SDN and NFV integration in generalized mobile network architecture," in Networks and Communications (EuCNC), 2015 European Conference on, June 2015, pp. 154-158.

[10] I. Ahmad et.all., "New Concepts for Traffic, Resource and Mobility Management in Software-Defined Mobile Networks", 2016 12th Annual Conference on Wireless On-demand Network Systems and Services (WONS).

[11] H. Koumaras, C. Sakkas, M. A. Kourtis, C. Xilouris, V. Koumaras and G. Gardikis, "Enabling agile video transcoding over SDN/NFV-enabled networks," 2016 International Conference on Telecommunications and Multimedia (TEMU), Heraklion, 2016, pp. 1-5.

[12] A. Kassler, L. Skorin-Kapov, O. Dobrijevic, M. Matijasevic and P. Dely, "Towards QoE-driven multimedia service negotiation and path optimization with software defined networking," Software, Telecommunications and Computer Networks (SoftCOM), 2012 20th International Conference on, Split, 2012, pp. 1-5.

[13] T. Hoßfeld et al., "An Economic Traffic Management Approach to Enable the TripleWin for Users, ISPs, and Overlay Providers," FIA Prague Book. Towards the Future Internet - A European Research Perspective: IOS Press Books Online, ISBN 978-1-60750-007-0, May 2009.

[14] M. R. Celenlioglu, S. B. Goger and H. A. Mantar, "An SDNbased energy-aware routing model for intra-domain networks," Software, Telecommunications and Computer Networks (SoftCOM), 2014 22nd International Conference on, Split, 2014, pp. 61-66.

[15] L. Skorin-Kapov, K. Ivesic, G. Aristomenopoulos, and S. Papavassiliou, "Approaches for utility-based QoE-driven optimization of network resource allocation for multimedia services," Data traffic monitoring and analysis, 2013, Springer Berlin Heidelberg, pp. 337-358

[16] F. Ongaro, E. Cerqueira, L. Foschini, A. Corradi and M. Gerla, "Enhancing the quality level support for real-time multimedia applications in software-defined networks," Computing, Networking and Communications (ICNC), 2015 International Conference on, Garden Grove, CA, 2015, pp. 505-509.

[17] H. J. Kim, D. G. Yun, H. S. Kim, K. S. Cho and S. G. Choi, ”QoE assessment model for video streaming service using QoS parameters in wiredwireless network," Advanced Communication Technology (ICACT), 2012 14th International Conference on, PyeongChang, 2012, pp. 459-464.

[18] Mininet, http://mininet.org/, [Accessed: 18- Oct- 2016].

[19] OpenDaylight Platform, https://www.opendaylight.org/, [Accessed: 18Oct- 2016].

[20] L. Skorin-Kapov and M. Matijasevic, "Modeling of a QoS Matching and Optimization Function for Multimedia Services in the NGN", in WiredWireless Multimedia Networks and Services Management, ser. Lecture Notes in Computer Science. Springer Berlin Heidelberg, 2009, vol.5842, pp. 55-68.

[21] OpenFlow Protocol, ONF, Available: https://www.opennetworking.org/sdn-resources/openflow, [Accessed: 18- Oct- 2016].

[22] Video LAN, http://www.videolan.org, [Accessed: 18- Oct- 2016].

[23] Emulating the delay, packet loss with NetEM, Available: https://wiki.linuxfoundation.org/networking/netem , [Accessed: 18Oct- 2016].

[24] M. Mu et al., "A Scalable User Fairness Model for Adaptive Video Streaming Over SDN-Assisted Future Networks," in IEEE Journal on Selected Areas in Communications, vol. 34, no. 8, pp. 2168-2184, Aug. 2016 